\title{
Quercetin reduces tendon adhesion in rat through suppression of oxidative stress
}

\author{
Yuan Liang ${ }^{1 \dagger}$, Keteng $\mathrm{Xu}^{1 \dagger}$, Pei Zhang ${ }^{2}$, Jiale Zhang ${ }^{1}$, Pengtao Chen ${ }^{1}$, Jinshan He${ }^{1}$, Yongchao Fang ${ }^{1}$, Yuelai Zhou', \\ Jingcheng Wang ${ }^{1^{*}}$ and Jianzhong Bai ${ }^{3^{*}}$
}

\begin{abstract}
Background: Tendon adhesion is one of the most common clinical problems, which poses a considerable challenge to orthopedics doctors. Quercetin (QUE) as a popular drug at present, it has various biological functions, including anti-inflammatory, anti-ischemic, anti-peroxidation, and antioxidant. The purpose of this study was to investigate the effect of quercetin on tendon adhesion and whether quercetin can inhibit oxidative stress.

Method: Thirty-six rats were randomly divided into three groups, including control group, low QUE (50 mg/kg/day) group, and high QUE (100 mg/kg/day) group. After 1 week, the levels of SOD, MDA and GPx were measured. The degree of tendon adhesion was assessed by macroscopic evaluation and histological evaluation. After 4 weeks. Besides, the pharmacological toxicity of quercetin to main organs were evaluated by histological analysis.

Results: The extent of superoxide dismutase (SOD) and glutathione peroxidase (GPx) of tendon tissue in high QUE group was significantly higher than those of low QUE group and control group. And the extent of malondialdehyde (MDA) of tendon tissue in high QUE group was significantly lower than that of low QUE group and control group. By macroscopic evaluation and histological analysis, the extent of tendon adhesion in high QUE group was lower than low QUE group and control group. However, there were no significant changes of the major organs through histological analysis.
\end{abstract}

Conclusions: Quercetin may be a good and safe strategy in preventing tendon adhesion. But further clinical research is needed before its recommendation in the prevention and treatment of tendon adhesion.

Keywords: Quercetin, Tendon adhesions, Oxidative stress

\section{Background}

Tendon adhesion is a critical clinical issue characterized by the limitation of tendon gliding, which poses a considerable challenge to orthopedic doctors $[1,2]$. Two pathways are considered to participate in tendon repair. The intrinsic repair pathway involves the internal repair

\footnotetext{
* Correspondence: wangjcyangzhou@163.com; jianzhongb@163.com

${ }^{\dagger}$ Yuan Liang and Keteng Xu contributed equally to this work.

'Department of Orthopedics, Clinical Medical College of Yangzhou

University, Subei People's Hospital, Nantong West Road 98, Yangzhou 225001, China

${ }^{3}$ Department of Orthopedics, The Second Affiliated Hospital of Bengbu Medical College, Bengbu 233000, China

Full list of author information is available at the end of the article
}

function of tenocytes, the extrinsic repair process involves the invasion of cells from the outside of tendon and finally promotes the formation of adhesion $[3,4]$. As the pathophysiological mechanism of tendon adhesion is not clear, the prevention and treatment of tendon adhesion is still facing challenges. Many methods have been used to prevent tendon adhesion, such as the improvement of surgical technology, the use of barriers, systemic or local application of drugs and chemicals. Although these methods can reduce adhesions to some extent, the residual or recurrent adhesions still need to be resolved [5-8]. 
Reactive oxygen species (ROS) as essential mediators of fibrogenesis, it is possible that ROS also play a role in the formation of tendon adhesion [9]. In addition, some studies have demonstrated that antioxidation strategies could reduce the extent of tendon adhesion by reducing ROS [10]. QUE is an abundant nature flavonoid which could be found in many fruits, vegetables, leaves and grains. It has been reported that QUE has the biological functions of anti-inflammation, anti-ischemia, antioxidation and anti-tumor [11-14]. Also, QUE is a strong antioxidant and radical scavenger [15-17]. Therefore, we considered it may be a functional drug to treat tendon adhesion, thus, we conducted rat models to figure out the effect of QUE on tendon adhesion.

\section{Methods}

\section{Ethical consent}

The animal experiments were carried out in accordance with the guide of the Clinical Medical College of Yangzhou University (Yangzhou, China).

\section{Chemicals}

Quercetin was purchased from Dalian Meilun Biological Technology Co., Ltd. (Dalian, China). SOD, GPx and MDA detection kits were purchased from Nanjing Jiancheng Biotechnology Limited (Nanjing, China).

\section{Animal preparation}

Thirty-six Male Wistar rats weighting 200 to 220 g were purchased from Yangzhou University (Yangzhou, China). The rats were enrolled equally into three groups, including control group, Low QUE (50 mg/kg/day) group, and High QUE (100 mg/kg/day) group. The doses were selected according to several published studies on the use of QUE in rats [18-21]. All rats were transferred to the laboratory 1 week before the study.

The animal model of tendon adhesion was established based on previous study [22]. Rats were anesthetized by intraperitoneal injection of $2 \%$ Pentobarbital Sodium (25 $\mathrm{mg} / \mathrm{kg}$ ) and fixed on the operating table. The right hind limb of each rat was shaved and scrubbed with povidone iodine. A longitudinal incision about $2 \mathrm{~cm}$ long were made on the inside of the plantar skin. Next, the subcutaneous tissue was dissected and the tendon was cut through by using $11 \#$ scalpels. The tendon was sutured with 5-0 nylon by modified Kessler suture technique. The skin incision was closed with 3-0 nylon. All rats were injected with penicillin $1,600,000 \mathrm{U} / \mathrm{kg}$ to prevent infection. The rats were kept in separate cages and were free to get food and water.

\section{Biochemical study}

Six rats of each group were randomly selected and sacrificed with overdose of pentobarbital at day 7 . Tendon tissues were stored at $-80^{\circ} \mathrm{C}$ until homogenized to analysis. The levels of malondialdehyde (MDA), superoxide dismutase (SOD) and glutathione peroxidase (GPx) activities were measured using different commercial assay kits.

\section{Macroscopic evaluation}

The other six rats of each group were killed with excessive pentobarbital 4 weeks after operation. The limbs were cut through the original incision. Two independent pathologists made blind observation on the experiment according to the previously reported methods: Level 1: no adhesion; Level 2: blunt peeling can separate adhesion; Level 3: sharp peeling is required, and no more than $50 \%$ of the adherent tissues are separated; level 4: sharp peeling can separate $51-97.5 \%$ of the adherent tissues; level 5: sharp peeling can separate more than $97.5 \%$ of the adherent tissues [23, 24]. When disagreement existed, it was resolved by consulting another pathologist.

\section{Histological analysis}

After macroscopic evaluation, tendon tissues were used for histological analysis. The samples were fixed in $4 \%$ paraform $24 \mathrm{~h}$, and then embedded in paraffin. Four successive transversal sections of four-micrometer were obtained. Two odd sections of each group were stained with hematoxylin and eosin ( $\mathrm{H} \& \mathrm{E})$ and the adhesions

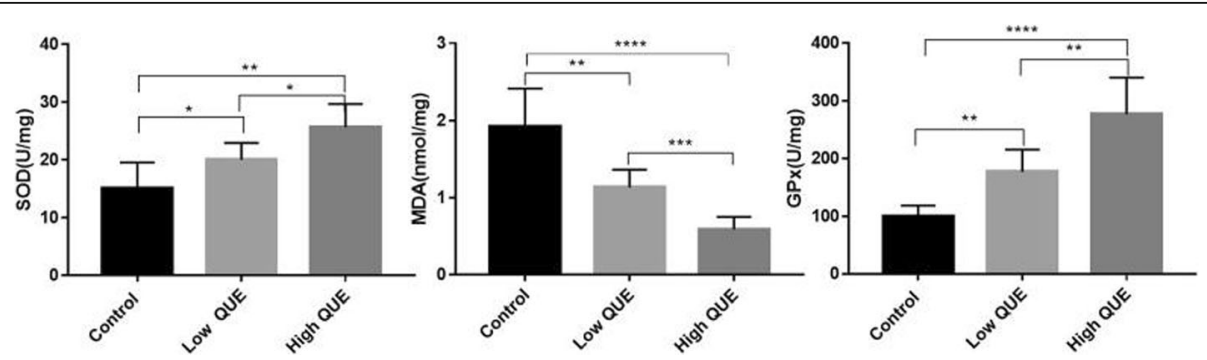

Fig. 1 The effect of QUE on oxidative stress-related products of tendon simples at day 7 after surgery. Six rats of each group were randomly selected. Quercetin decreased levels of MDA and elevated activity of SOD and GSH-Px. ${ }^{*} P<0.05 ;{ }^{* *} P<0.01 ;{ }^{* * *} P<0.001$. Low QUE; 50 mg/kg/day; High QUE, $100 \mathrm{mg} / \mathrm{kg} / \mathrm{day}$ 
Table 1 Gross adhesion scores in different groups

\begin{tabular}{llllll}
\hline Group & Grade & & & & \\
\cline { 2 - 6 } & $\mathbf{1}$ & $\mathbf{2}$ & $\mathbf{3}$ & $\mathbf{4}$ & $\mathbf{5}$ \\
\hline control & & & 2 & 3 & 1 \\
Quercetin $(50 \mathrm{mg} / \mathrm{kg})$ & & 3 & 2 & 1 & \\
Quercetin $(100 \mathrm{mg} / \mathrm{kg})$ & 1 & 4 & 1 & & \\
\hline
\end{tabular}

were evaluated under the light microscope with the magnification $\times 200$. Two even sections of each group were stained with Masson's trichrome and the collagen density of adhesion tissue was evaluated at 200× magnification. All sections for an individual case were counted blinded to the group assignment.

\section{Statistical analysis}

The data were analyzed using SPSS 22.0. The significance of differences was calculated by using one-way analysis of variance (ANOVA) followed by Tukey for multiple comparisons. $P<0.05$ was considered as statistically significant.

\section{Results}

The surgery was well tolerated by all rats. There was no wound infection and mortality during the experiment.

\section{Effect of treatment of QUE on antioxidant enzyme activities}

As shown in Fig. 1, QUE significantly increased the activities of SOD (Control: 15.02 \pm 1.844 , Low QUE: $20 \pm$ 1.202, High QUE: $25.68 \pm 1.643$ ) and GPx (Control: $99.96 \pm 7.744$, Low QUE: $176.9 \pm 15.81$, High QUE: $277 \pm 25.61$ ). The MDA levels were significantly lower in the QUE groups (Control: $1.92 \pm 0.203$, Low QUE: $1.13 \pm 0.09448$, High QUE: $0.5917 \pm 0.06524)$. Besides, our results showed the antioxidant capacity of QUE was dose-dependent.

\section{Macroscopic evaluation of tendon adhesion}

Macroscopic observation showed that there was weak or moderate adhesion in quercetin treated group. However, in control group, thick fibrous adhesions were observed.
The degree of tendon adhesions was evaluated according to the above method (Table 1).

\section{Effect of QUE on tendon adhesion in histological analysis} In control group, dense scar adhesions were found around the surgical areas. In $50 \mathrm{mg} / \mathrm{kg}$ quercetin-treated groups, mild scar tissues were observed around the surgical compared with those of control group. However, no or loose fibrous adhesion tissue were observed in $100 \mathrm{mg} / \mathrm{kg}$ quercetin-treated group (Fig. 2).

\section{Effect of QUE on collagen density of tendon}

In Masson's trichrome staining, collagen density of tendon adhesion tissue in quercetin-treated groups was coincidence with HE staining. The collagen density of tendon tissue in control group was dense. However, the collagen density was weak in $100 \mathrm{mg} / \mathrm{kg}$ quercetintreated groups, which revealed decrease compared with those in $50 \mathrm{mg} / \mathrm{kg}$ quercetin-treated groups. Moreover, the collagen density was moderate in $50 \mathrm{mg} / \mathrm{kg}$ quercetin-treated groups, which was also revealed decrease compared with that in control group (Fig. 3).

\section{Pilot toxicity study}

To evaluate the potential toxicity of QUE in vivo. The toxicity to major organs (Heart, liver, spleen, lung and kidney) was investigated by $\mathrm{HE}$ staining (Fig. 4). The histological analysis indicated that QUE did not show any distinct changes of the major organs at the end of this study. All these results demonstrated that our developed therapeutic strategy was effective and safe.

\section{Discussion}

Our study demonstrated that QUE could increase SOD and GPX, and reduce MDA in a dose-dependent manner. Besides, the degree of tendon adhesion in high QUE group was lower than low QUE group and control group. Furthermore, the histological analysis of main organs showed that quercetin has no obvious toxicity.

Tendon adhesion mostly occurs after tendon surgery and joint immobilization. Several mechanisms have been implicated in the modulation of adhesion formation,

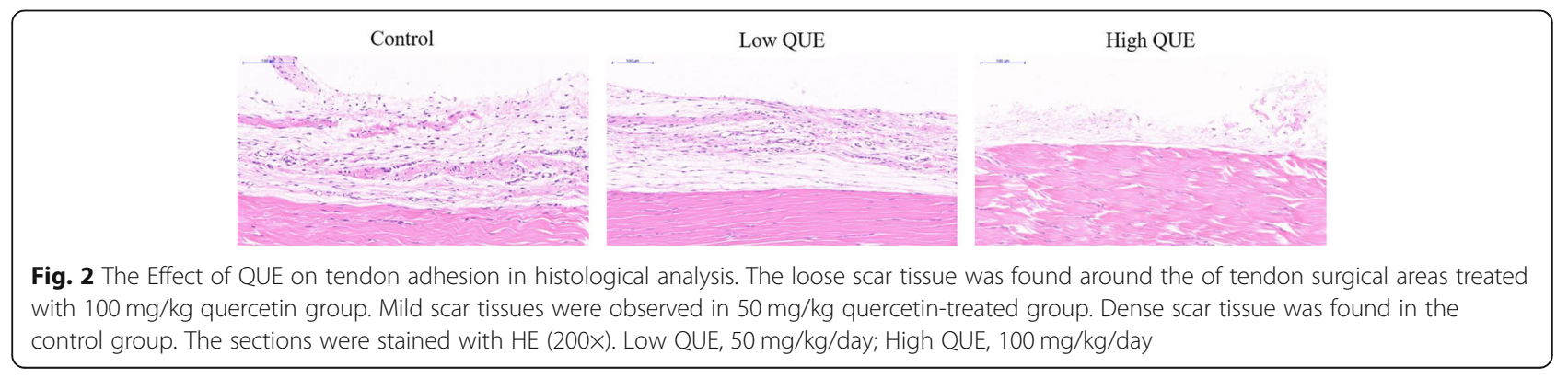



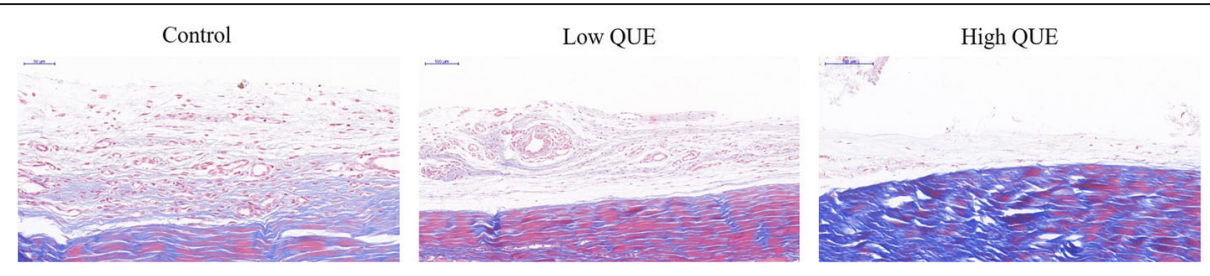

Fig. 3 The effect of QUE on tendon collagen density. The collagen tissues show blue in the section with Masson's trichrome staining under the light microscope (200X). QUE could reduce collagen synthesis and fibrosis. The density of collagen tissue in $100 \mathrm{mg} / \mathrm{kg}$ quercetin-treated group seemed to be lower than those in $50 \mathrm{mg} / \mathrm{kg}$ quercetin-treated group and control group. Low QUE, 50 mg/kg/day; High QUE, 100 mg/kg/day

including the regulation of the TGF- $\beta$ signaling pathway, oxidative stress, and the inflammatory response [3, 25].

ROS is mainly produced by mitochondria, which plays a dual role in the process of cell physiology. The low level of ROS can regulate specific signal pathways, and too many ROS participate in the pathogenesis of many fibrotic diseases such as tendon adhesion [26-28]. It can be attenuated by various antioxidant enzymes such as SOD and GPx, which could indirectly reflect the oxidative state [29, 30]. Malondialdehyde (MDA, a marker of lipid peroxidation) is usually used as a biomarker of oxidative damage [31]. Increased MDA production and oxidative stress are able to accelerate the accumulation of ECM components and the proliferation of interstitial fibroblasts. In addition, more and more evidences have strongly implicated MDA production and oxidative stress in the pathological of fibrosis [32]. Quercetin is one of the most prevalent plant flavonoids contained in many fruits and vegetables. Within the flavonoid family, quercetin is the most potent scavenger of ROS
[33, 34]. Previous studies reported that quercetin could ameliorate pulmonary fibrosis, renal fibrosis, liver fibrosis [35-37].

In current study, we collected tendon samples to measure MDA, SOD and GPx after 1 week of surgery. The results showed that QUE significantly increased the activities of SOD (Control: 15.02 \pm 1.844 , Low QUE: $20 \pm 1.202$, High QUE: $25.68 \pm 1.643$ ) and GPx (Control: $99.96 \pm 7.744$, Low QUE: $176.9 \pm 15.81$, High QUE: $277 \pm 25.61$ ). While, the MDA levels were significantly decreased in the QUE groups (Control: 1.92 \pm 0.203 , Low QUE: $1.13 \pm 0.09448$, High QUE: $0.5917 \pm 0.06524$ ), and the effect was dose-dependent. To further the research, we made macroscopic evaluation and histological analysis of the tendon 4 weeks after the operation. Compared with the control group, the adhesion of tendon in quercetin group was relieved obviously, and the effect of reducing adhesion was better with the increase of quercetin dose, which is consistence with the results of measurement of SOD, GPx, fMDA.

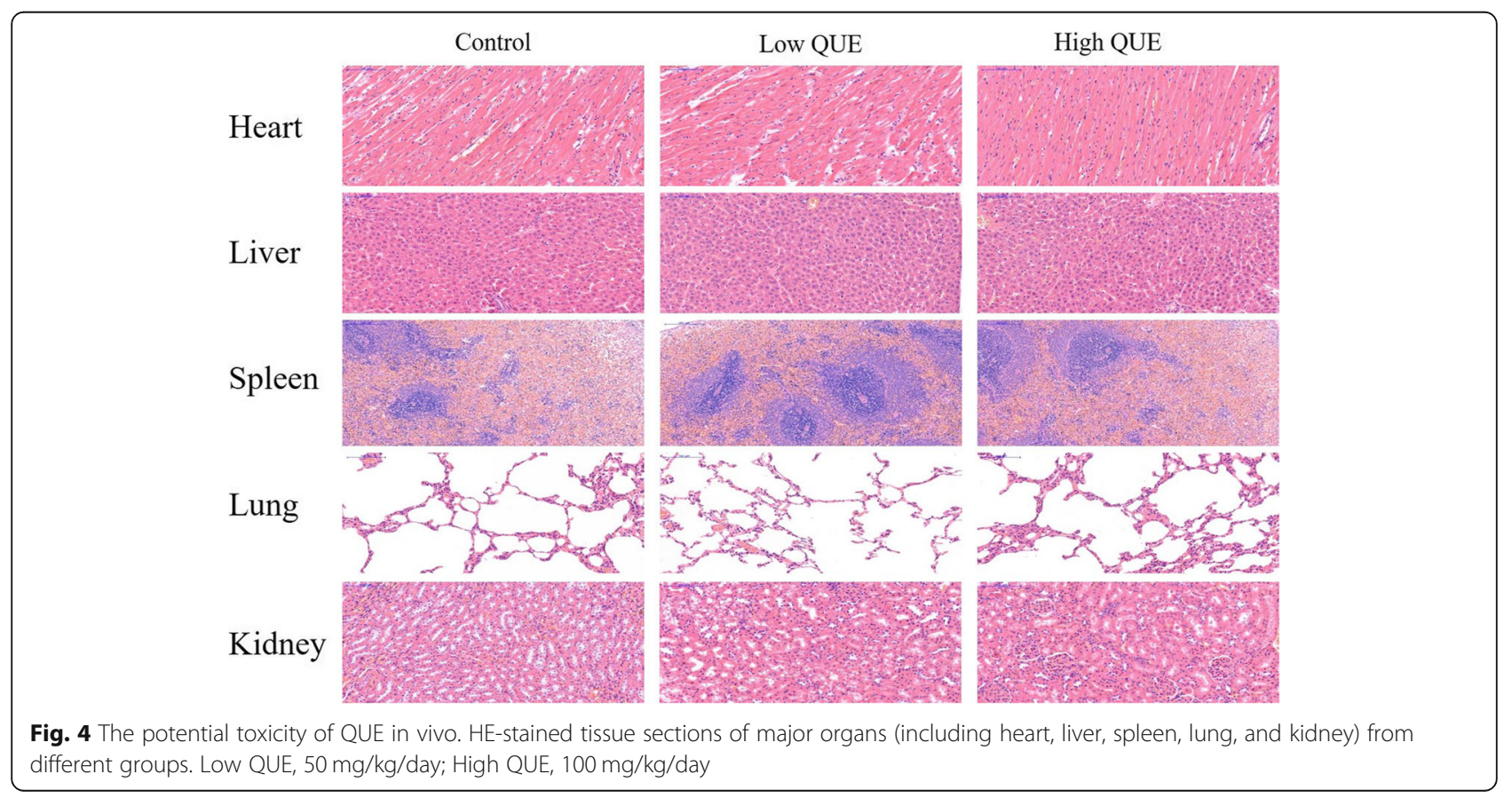


Interestingly, we did not see any significant adverse effects in terms of signs of infection and wound healing. Considering the possibility of the potential toxicity for the major organs, we also investigated the major organs by HE staining. Fortunately, histological analysis also demonstrated that quercetin didn't show any pilot toxicity.

\section{Conclusion}

Quercetin may be a good and safe strategy in preventing tendon adhesion. But further clinical research is needed before its recommendation in the prevention and treatment of tendon adhesion.

\section{Abbreviations \\ QUE: Quercetin; SOD: Superoxide dismutase; GPx: Glutathione peroxidase; MDA: Malondialdehyde; ROS: Reactive oxygen species}

\section{Acknowledgments}

Not applicable.

\section{Authors' contributions}

WJ and BJ conceived of the design of the study. ZP and CP participated in animal preparation. ZJ and ZY participated in data collection. FY and HJ performed the statistical analysis. LY and XK finished the manuscript. All authors read and approved the final manuscript.

\section{Funding}

This study was supported by the National Natural Science Foundation of China (No: 81772332); Jiangsu medical innovation team project (No: CXTDB2017004) in analysis and interpretation of data.

\section{Availability of data and materials}

The datasets used and/or analyzed during the current study are available from the corresponding author upon reasonable request.

\section{Ethics approval}

The animal experiments were carried out in accordance with the guide of the Clinical Medical College of Yangzhou University (Yangzhou, China).

\section{Consent for publication}

Not applicable.

\section{Competing interests}

The authors declare that they have no competing interests.

\section{Author details \\ 'Department of Orthopedics, Clinical Medical College of Yangzhou University, Subei People's Hospital, Nantong West Road 98, Yangzhou 225001, China. ${ }^{2}$ Department of Orthopedics, The Second Xiangya Hospital of Central South University, Changsha 410011, Hunan, China. ${ }^{3}$ Department of Orthopedics, The Second Affiliated Hospital of Bengbu Medical College, Bengbu 233000, China}

Received: 26 March 2020 Accepted: 31 August 2020

Published online: 11 September 2020

\section{References}

1. de Jong JP, Nguyen JT, Sonnema AJ, Nguyen EC, Amadio PC, Moran SL. The incidence of acute traumatic tendon injuries in the hand and wrist: a 10year population-based study. Clin Orthop Surg. 2014;6(2):196-202.

2. Legrand A, Kaufman Y, Long C, Fox PM. Molecular biology of flexor tendon healing in relation to reduction of tendon adhesions. J Hand Surg Am. 2017:42(9):722-6.

3. Fatemi MJ, Shirani S, Sobhani R, Lebaschi AH, Gharegozlou MJ, Bagheri T, Pedram M, Saberi M, Araghi S, Fatemi MA. Prevention of Peritendinous adhesion formation after the flexor tendon surgery in rabbits: a comparative study between use of local interferon-alpha, interferon-beta, and 5fluorouracil. Ann Plast Surg. 2018;80(2):171-5.

4. Graham JG, Wang ML, Rivlin M, Beredjiklian PK. Biologic and mechanical aspects of tendon fibrosis after injury and repair. Connect Tissue Res. 2019; 60(1):10-20.

5. Tosun HB, Gumustas $S A$, Kom M, Uludag A, Serbest $S$, Eroksuz $Y$. The effect of sodium hyaluronate plus sodium chondroitin sulfate solution on Peritendinous adhesion and tendon healing: An experimental study. Balkan Med J. 2016;33(3):258-66.

6. Lee YW, Fu SC, Mok TY, Chan KM, Hung LK. Local administration of Trolox, a vitamin $\mathrm{E}$ analog, reduced tendon adhesion in a chicken model of flexor digitorum profundus tendon injury. J Orthop Translat. 2017;10:102-7.

7. Liao JCY, He M, Gan AWT, Wen F, Tan LP, Chong AKS. The effects of bifunctional anti-adhesion scaffolds on flexor tendon healing in a rabbit model. J Biomed Mater Res B Appl Biomater. 2018;106(7):2605-14.

8. Strick MJ, Filan SL, Hile M, McKenzie C, Walsh WR, Tonkin MA. Adhesion formation after flexor tendon repair: comparison of two- and four-strand repair without epitendinous suture. Hand Surg. 2005;10(2-3):193-7.

9. Kisseleva T, Brenner DA. Mechanisms of fibrogenesis. Exp Biol Med (Maywood). 2008;233(2):109-22.

10. Hung LK, Fu SC, Lee YW, Mok TY, Chan KM. Local vitamin-C injection reduced tendon adhesion in a chicken model of flexor digitorum profundus tendon injury. J Bone Joint Surg Am. 2013;95(7):e41.

11. Li G, Shen X, Wei Y, Si X, Deng X, Wang J. Quercetin reduces Streptococcus suis virulence by inhibiting suilysin activity and inflammation. Int Immunopharmacol. 2019;69:71-8.

12. Ekinci Akdemir FN, Gulcin I, Karagoz B, Soslu R. Quercetin protects rat skeletal muscle from ischemia reperfusion injury. J Enzyme Inhib Med Chem. 2016;31(sup2):162-6.

13. Sadhukhan P, Kundu M, Chatterjee S, Ghosh N, Manna P, Das J, Sil PC Targeted delivery of quercetin via pH-responsive zinc oxide nanoparticles for breast cancer therapy. Mater Sci Eng C Mater Biol Appl. 2019;100:129-40.

14. Feng $K$, Chen Z, Pengcheng L, Zhang S, Wang X. Quercetin attenuates oxidative stress-induced apoptosis via SIRT1/AMPK-mediated inhibition of ER stress in rat chondrocytes and prevents the progression of osteoarthritis in a rat model. J Cell Physiol. 2019;234(10):18192-205.

15. Young JF, Nielsen SE, Haraldsdottir J, Daneshvar B, Lauridsen ST, Knuthsen P. Crozier A, Sandstrom B, Dragsted LO. Effect of fruit juice intake on urinary quercetin excretion and biomarkers of antioxidative status. Am J Clin Nutr. 1999:69(1):87-94.

16. Lamson DW, Brignall MS. Antioxidants and cancer, part 3: quercetin. Altern Med Rev. 2000:5(3):196-208.

17. Horton JA, Li F, Chung EJ, Hudak K, White A, Krausz K, Gonzalez F, Citrin D. Quercetin inhibits radiation-induced skin fibrosis. Radiat Res. 2013;180(2): 205-15.

18. Verma R, Kushwah L, Gohel D, Patel MV, Marvania T, Balakrishnan S. Evaluating the Ameliorative Potential of Quercetin against the Bleomycin-Induced Pulmonary Fibrosis in Wistar Rats. Pulm Med. 2013; 2013:921724.

19. Lu Q, Ji X, Zhou Y, Yao X, Liu Y, Zhang F, Yin X. Quercetin inhibits the mTORC1/p70S6K signaling-mediated renal tubular epithelial-mesenchymal transition and renal fibrosis in diabetic nephropathy. Pharmacol Res. 2015; 99:237-47.

20. Liu Y, Dai E, Yang J. Quercetin suppresses glomerulosclerosis and TGF- $\beta$ signaling in a rat model. Mol Med Rep. 2019;19(6):4589-96.

21. Liu S, Tian L, Chai G, Wen B, Wang B. Targeting heme oxygenase-1 by quercetin ameliorates alcohol-induced acute liver injury via inhibiting NLRP3 inflammasome activation. Food Funct. 2018;9(8):4184-93.

22. Tang XM, Dai J, Sun HL. Thermal pretreatment promotes the protective effect of HSP70 against tendon adhesion in tendon healing by increasing HSP70 expression. Mol Med Rep. 2019;20(1):205-15.

23. Zheng W, Song J, Zhang Y, Chen S, Ruan H, Fan C. Metformin prevents peritendinous fibrosis by inhibiting transforming growth factor-beta signaling. Oncotarget. 2017:8(60):101784-94.

24. Jiang S, Zhao X, Chen S, Pan G, Song J, He N, Li F, Cui W, Fan C. Downregulating ERK1/2 and SMAD2/3 phosphorylation by physical barrier of celecoxib-loaded electrospun fibrous membranes prevents tendon adhesions. Biomaterials. 2014;35(37):9920-9.

25. Zheng W, Qian Y, Chen S, Ruan H, Fan C. Rapamycin protects against Peritendinous fibrosis through activation of autophagy. Front Pharmacol. 2018;9:402. 
26. ten Raa S, van den Tol MP, Sluiter W, Hofland LJ, van Eijck CH, Jeekel H. The role of neutrophils and oxygen free radicals in post-operative adhesions. J Surg Res. 2006;136(1):45-52.

27. Wang D, Yan Z, Bu L, An C, Deng B, Zhang J, Rao J, Cheng L, Zhang J, Zhang B, Xie J. Protective effect of peptide DR8 on bleomycin-induced pulmonary fibrosis by regulating the TGF-beta/MAPK signaling pathway and oxidative stress. Toxicol Appl Pharmacol. 2019;382:114703.

28. Xiao X, Yuan Q, Chen Y, Huang Z, Fang X, Zhang H, Peng L, Xiao P. LncRNA ENST00000453774.1 contributes to oxidative stress defense dependent on autophagy mediation to reduce extracellular matrix and alleviate renal fibrosis. J Cell Physiol. 2019;234(6):9130-43.

29. Wang SQ, Li D, Yuan Y. Long-term moderate intensity exercise alleviates myocardial fibrosis in type 2 diabetic rats via inhibitions of oxidative stress and TGF-beta1/Smad pathway. J Physiol Sci. 2019;69(6):861-73.

30. Li C, Sun X, Li A, Mo M, Zhao Z. S-Allylmercaptocysteine attenuates Bleomycin-induced pulmonary fibrosis in mice via suppressing TGF-betal/ Smad and oxidative stress pathways. Int Immunopharmacol. 2019;79:106110.

31. Zhao Q, Yang F, Meng L, et al. Lycopene attenuates chronic prostatitis/ chronic pelvic pain syndrome by inhibiting oxidative stress and inflammation via the interaction of NF-KB, MAPKs, and Nrf2 signaling pathways in rats. Andrology. 2020;8(3):747-55.

32. Sun J, Wu Y, Long C, He P, Gu J, Yang L, Liang Y, Wang Y. Anthocyanins isolated from blueberry ameliorates CCI4 induced liver fibrosis by modulation of oxidative stress, inflammation and stellate cell activation in mice. Food Chem Toxicol. 2018;120:491-9.

33. Kashyap D, Garg VK, Tuli HS, Yerer MB, Sak K, Sharma AK, Kumar M, Aggarwal V, Sandhu SS. Fisetin and Quercetin: Promising Flavonoids with Chemopreventive Potential. Biomolecules. 2019;9(5):174.

34. Boots AW, Haenen GR, Bast A. Health effects of quercetin: from antioxidant to nutraceutical. Eur J Pharmacol. 2008;585(2-3):325-37.

35. Zhang $X$, Cai $Y$, Zhang W, Chen $X$. Quercetin ameliorates pulmonary fibrosis by inhibiting SphK1/S1P signaling. Biochem Cell Biol. 2018;96(6):742-51.

36. Ren J, Li J, Liu X, Feng Y, Gui Y, Yang J, He W, Dai C. Quercetin inhibits fibroblast activation and kidney fibrosis involving the suppression of mammalian target of Rapamycin and beta-catenin signaling. Sci Rep. 2016; 6:23968.

37. Li X, Jin Q, Yao Q, Xu B, Li L, Zhang S, Tu C. The flavonoid Quercetin ameliorates liver inflammation and fibrosis by regulating hepatic macrophages activation and polarization in mice. Front Pharmacol. 2018;9:72.

\section{Publisher's Note}

Springer Nature remains neutral with regard to jurisdictional claims in published maps and institutional affiliations.

Ready to submit your research? Choose BMC and benefit from:

- fast, convenient online submission

- thorough peer review by experienced researchers in your field

- rapid publication on acceptance

- support for research data, including large and complex data types

- gold Open Access which fosters wider collaboration and increased citations

- maximum visibility for your research: over $100 \mathrm{M}$ website views per year

At $\mathrm{BMC}$, research is always in progress.

Learn more biomedcentral.com/submissions 